\title{
A case of atypical sporadic hemiplegic migraine associated with PFO and hypoplasia of vertebro-basilar system
}

\author{
Filippo Brighina $\cdot$ Giacomo Gurgone $\cdot$ \\ Rosa Maria Gaglio · Antonio Palermo • \\ Giuseppe Cosentino $\cdot$ Brigida Fierro
}

Received: 16 March 2009/ Accepted: 10 April 2009/Published online: 7 May 2009

(C) Springer-Verlag 2009

\begin{abstract}
We describe the case of a patient with atypical hemiplegic migraine and associated basilar symptoms, where a large patent foramen ovale (PFO) and hypoplasia of basilar artery were found. The longer period of 4-year remission of the headache attacks was coincident with the percutaneous PFO closure. When 5 years after, hemiplegic migraine attacks relapsed, with more relevant basilar symptoms, a mild re-opening of PFO was found. The atypical presentation of attacks with basilar symptoms and prolonged hemiplegia does not strictly fit the diagnostic criteria of ICHD-II.
\end{abstract}

Keywords Migraine with aura - Hemiplegic migraine . PFO - Basilar type migraine - Vertebro-basilar system hypoplasia

\section{Introduction}

Particular presentations of migraine aura include hemiplegic migraine, in which aura consists of fully reversible motor weakness together with visual, sensory or speech disturbances, and basilar type migraine (BTM). This is a rare form of migraine that could present with a dramatic clinical picture, mimicking other severe neurological disorders. This could be particularly puzzling when complications of migraine as "persistent aura without infarction" occur [1]. BTM presents symptoms referred to posterior

F. Brighina $(\bowtie) \cdot$ A. Palermo $\cdot$ G. Cosentino $~ B$ B. Fierro

Department of Clinical Neurosciences,

University of Palermo, Palermo, Italy

e-mail: fbrighina@unipa.it

G. Gurgone - R. M. Gaglio

Ospedale di Agrigento, Agrigento, Italy cerebral circulation involvement: dysarthria, vertigo, tinnitus, hypoacusia, diplopia, ataxias, bilateral sensitive or visual deficits, and decreased level of consciousness.

According to symptoms of typical aura (ICHD-II), in both conditions aura lasts from 5 to $60 \mathrm{~min}$, except for motor weakness (in hemiplegic migraine) that can last till $24 \mathrm{~h}$ [2]. Patent foramen ovale (PFO) has been reported to have an elevated prevalence in patients suffering from migraine with aura (MA) [3]. The possibility that right-toleft shunt may be a "trigger" in the genesis of aura has also been suggested [4], even if it is yet debated whether the primary mechanisms of migraine are vascular or neurogenic. Recent reports concerning the relationship between MA and PFO included patients with motor weakness also [5, 6]. In particular, in the report by Anzola et al. [6] 12\% of patients with right-to-left shunt exhibited motor symptoms. Here, we describe a peculiar case of a patient with features "suggestive" for hemiplegic migraine (with prolonged hemiplegia and basilar symptoms) associated with a basilar artery hypoplasia and in which a large PFO was found and surgically treated by percutaneous closure.

\section{Case report}

A 35-year-old male patient came to our attention 5-years ago because of acute motor weakness in the left side of his body, together with a slurred speech, dizziness, and mild headache. These symptoms underwent a complete resolution after 3 weeks. The patient underwent the first episode 2-years before, when acutely he complained of same symptoms (that lasted for 3 weeks). He was a non smoker, and had a familial and medical history negative for nervous system disorders (including migraine). Hematological screening for thrombophilic risk factors (LAC, thrombocythemia, etc.), brain CT, 
Fig. 1 a RM scan with T2FLAIR (Fluid-AttenuatedInversion-Recovery) technique, which shows a normal cerebral parenchyma. b a DWI-RMI scan, performed using the ADC (Apparent Diffusion Coefficient) protocol, which shows no alteration of signal
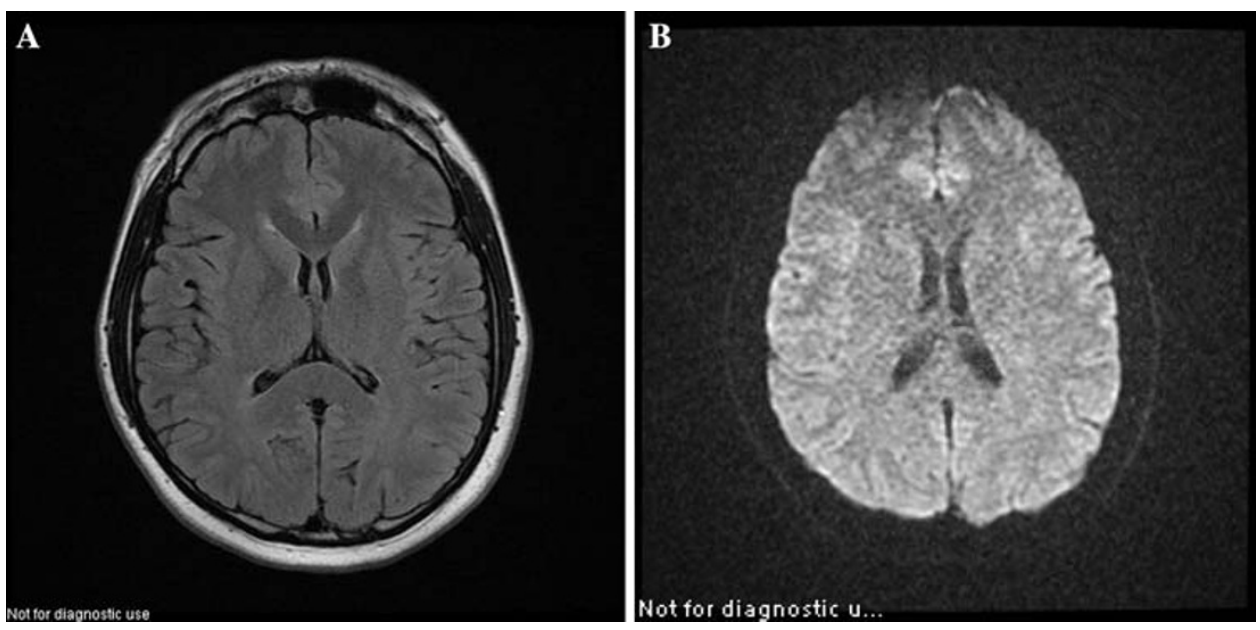

and MR imaging (including DWI scan) as in Fig. 1a, b were all normal. No pathological enhancements with gadolinium were observed.

A mild hypertension (150/90 $\mathrm{mm} \mathrm{Hg}$ ) was found, and a trans-esophageal-echocardiography (TEE) showed a PFO with a large right-to-left shunt.

On such basis a presumptive "cerebro-vascular" accident was diagnosed. Antiplatelet inhibitor and anti-hypertensive drug (ACE-inhibitor) were prescribed, and the patient was subsequently referred to an interventional center for trans-catheter PFO closure. A further diagnostic work-up with magnetic resonance angiography showed a marked hypoplasia of vertebro-basilar system, ruling out

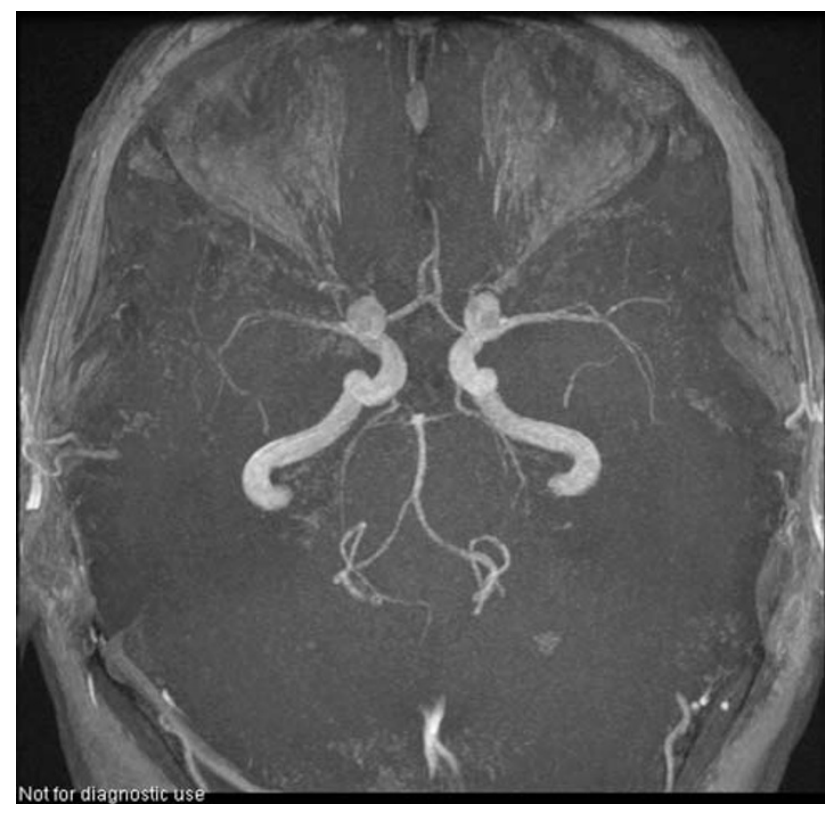

Fig. 2 An angiographic RM scan (using the time of flight technique, TOF, with digital three-dimensional reconstruction and planar projection on a T1-weighted sequence), which shows the stenosis of the basilar artery other abnormalities of cerebral vessels and also deep venous thrombosis (Fig. 2).

After the PFO closure, our patient experienced a full well-being until the summer 2007, when he was referred to an emergency area for a new episode of acute weakness of the left side, prevalent in the arm and dysarthria, that completely recovered after $72 \mathrm{~h}$. High blood pressure values were found and the $\mathrm{CT}$ scan of brain did not reveal ischemic or hemorrhagic acute lesion. Then a MR imaging and a MR angiography were obtained, confirming the basilar artery hypoplasia, previously observed. The patient underwent a new TEE, which showed a "re-open" PFO with a "mild" right-to-left shunt.

The patient was hospitalized in a medical university center, and was evaluated for a new heart surgical treatment. After admission, he experienced a new episode with weakness of his left body, and again difficulty in the speech and dizziness. Neuro-radiological studies did not show any further abnormality. Clinical picture gradually subsided within few days, and he was discharged with diagnosis of "possible hemiplegic migraine associated to basilar symptoms and stenosis of the basilar artery". Based on the entity of the shunt, surgical PFO closure was not considered, and anticoagulant therapy (warfarin) was prescribed.

One month later, the patient came back to our observation because of a severe and abrupt dizziness with unsteady gait, suddenly followed by difficulty of speech articulation, diplopia, paresthesia, progressive weakness of the left side, nausea, and vomiting. In the next hours the clinical picture worsened evolving to anarthria and left hemiplegia.

This condition lasted for almost $5 \mathrm{~h}$; then the patient partially recovered, complaining of a mild throbbing occipital headache. Complete recovery of speech occurred after 1 week persisting with only slight hyposthenia of the left arm. CT scan of the brain remained negative. This was the last episode until now. 


\section{Discussion}

In our case some of the clinical features of the aura (dysarthria, diplopia, dizziness etc.) during attacks were expression of brainstem dysfunction, so fulfilling the criteria for diagnosis of basilar-type migraine (ICHD-II) [2]. However, the presence of the motor weakness (consistent across attacks) and the length of the aura (more than 1 week), made the inclusion in this nosographic category inadequate. Indeed, according to the last International Classification of Headache Disorders 2nd Edition revision (ICHD-II) [2], basilar-type migraine should be diagnosed only when no motor weakness occurs; therefore the diagnosis of sporadic hemiplegic migraine (no familial history of migraine in our patient) could appear more appropriated, even if the lack of visual or sensory symptoms makes diagnosis very puzzling. Moreover the slow resolution of the motor aura that went beyond $24 \mathrm{~h}$ could be interpreted as "persistent aura without infarction" and the attacks classifiable as "probable migraine with aura". Namely, repeated neuroimaging examination excluded ischemic damage or other cerebral pathologies underlying symptoms.

Familial hemiplegic migraine has basilar-type symptoms in $60 \%$ of cases and recently a novel mutation in the ATP1A2 gene $(\mathrm{R} 548 \mathrm{H})$ in members of a family with BTM has been detected, suggesting that BTM and familial hemiplegic migraine (FHM) may be allelic disorders [7, 8]. The most intriguing aspect in our case was the presence of a large PFO. Several data in literature indicate an increased incidence of PFO in MA patients compared with general population [9, 10]. A closure of PFO in patients with transient cerebral ischemia suffering from MA induced an improvement of MA episodes [11]. However, the role of PFO in migraine pathogenesis remains to be established and, based on the recently published MIST trial data [12], there is no sufficient evidence that recurrence of migraine is improved by PFO closure.

On such basis, in the present case the 4-year persisting remission of migraine cannot be convincingly attributed to the PFO closure. Similarly, taking the natural history of hemiplegic migraine also into account, notoriously difficult to predict, it is not obvious to establish a causal link between PFO re-opening and attack relapsing. Nevertheless, it cannot be excluded that, in our patient, PFO plays a role, at least as cofactor, in the complex pathogenetic mechanisms of migraine.

Finally, it is interesting to note that our patient also had a marked hypoplasia of the basilar artery. There is a causal relationship between these conditions or is the association just coincidental?

It is worth noting that in our patient, symptoms of brainstem dysfunction were consistent across all attacks.
Since the original description of Birkerstaff [13] that attributed the brainstem dysfunction in BTM to the "constriction" of the basilar artery, "reversible vertebro-basilar ischemia" has been described in patients with BTM and FHM [14, 15]. Recently, it has been reported in a case of basilar artery fenestration and hypoplasia of the right posterior cerebral artery in a woman with basilar-type migraine [16]. About the possible role of basilar artery stenosis, it is to be noted that a higher frequency of hypoplastic vertebral artery (VA) was found in MA [17]. The authors suggested that hypoplasia of VA may be an additional factor leading to hypoperfusion in the posterior circulation during the aura phase. More recently Chuang et al. hypothesized that VA hypoplasia contributes to migraine trough complex neurovascular pathways rather than trough its low-flow volume [18].

Although vascular mechanisms are recently considered less relevant in migraine pathogenesis, vascular factors could play a role in some phases of the cascade underlying the migraine attack. The question remains open.

\section{Conflict of interest None.}

\section{References}

1. Selby G (1983) Migraine and its variants. ADIS press, Sidney

2. Headache Classification Committee of the International Headache Society (2004) Classification and diagnostic criteria for headache disorders, cranial neuralgias, and facial pain. Cephalalgia 24(Supp 1):1-160

3. Sztajzel R, Genoud D, Roth S, Mermillod B, Le Floch-Rohr J (2003) Patent foramen ovale, a possible cause of symptomatic migraine: a study of 74 with acute ischemic stroke. Cerebrovasc Dis 13:102-106. doi:10.1159/000047758

4. Wilmshurst P, Nigthingale S (2001) Relationship between migraine and cardiac and pulmonary right-to-left shunts. Clin Sci 100:215-220. doi:10.1042/CS20000231

5. Domitrz I, Miezskowski J, Kaminska A (2007) Relationship between migraine and patent foramen ovale: a study of 121 patients with migraine. Headache 47(9):1311-1318. doi:10.1111/ j.1526-4610.2006.00724.x

6. Anzola GP, Meneghetti G, Zanferrrari C, Adami A, Dinia L, Del Sette M, SAM Study Group (2008) Is migraine associated with a right-to-left shunt a separate disease? Results of the SAM study. Cephalalgia 8(4):360-366. doi:10.1111/j.1468-2982.2008. 01539.x

7. Ambrosini A, D'Onofrio M, Grieco GS, Di Mambro A, Montagna G, Fortini D, Nicoletti F, Nappi G, Sances G, Schoenen J, Buzzi MG, Santorelli FM, Pierelli F (2005) Familial basilar migraine associated with a new mutation in the ATP1A2 gene. Neurology 65:1826-1828. doi:10.1212/01.wnl.0000187072. 71931.c0

8. Thomsen LL, Eriksen MK, Roemer SF, Andersen I, Olesen J, Russell MB (2002) A population based study of familial hemiplegic migraine suggests revised diagnostic criteria. Brain 125:1379-1391. doi:10.1093/brain/awf132

9. Carol-Artal FJ, da Silverira Ribeiro L, Braga H, Kummer W, Mesquita HM, Vargas AP (2006) Prevalence of patent foramen ovale in migraine patients with and without aura compared with 
stroke patients. A transcranial Doppler study. Cephalalgia 26:934-939. doi:10.1111/j.1468-2982.2006.01156.x

10. Wilmshurt P, Nightingale S, Pearson M, Morrison L, Walsh K (2006) Relation of atrial shunts to migraine in patients with ischemic stroke and peripheral emboli. Am J Cardiol 98:831-833. doi:10.1016/j.amjcard.2006.03.070

11. Onorato E, Melzi G, Casilli F, Pedon L, Rigatelli G, Carrozza A, Maiolino P, Zanchetta M, Morandi E, Angeli S, Anzola GP (2003) Patent foramen ovale with paradoxical embolism: midterm results of trans-catheter closure in 256 patients. J Interv Cardiol 16:43-50. doi:10.1046/j.1540-8183.2003.08002.x

12. Dowson A, Mullen MJ, Peatfield R, Muir K, Khan AA, Wells C, Lipscombe SL, Rees T, De Giovanni JV, Morrison WL, Hildick-Smith D, Elrington G, Hillis WS, Malik IS, Rickards A (2008) Migraine Intervention with STARFlex Technology (MIST) trial: a prospective, multicenter, double-blind, shamcontrolled trial to evaluate the effectiveness of patent foramen ovale closure with STARFlex septal repair implant to resolve refractory migraine headache. Circulation 117:1397-1404. doi:10.1161/CIRCULATIONAHA.107.727271
13. Bickerstaff ER (1961) Basilar artery migraine. Lancet 1:15-17. doi:10.1016/S0140-6736(61)92184-5

14. Caplan LR (1991) Migraine and vertebro-basilar ischemia. Neurology 41:55-61

15. Seto H, Shimizu M, Futatsuya R (1994) Basilar artery migraine: reversible ischemia demonstrated by Tc-99m HMPAO brain SPECT. Clin Nucl Med 19:215-218. doi:10.1097/00003072199403000-00010

16. Morelli N, Gori S, Mancuso M, Murri L (2007) Basilar artery fenestration in a woman with basilar-type migraine. Neurol Sci 28:116-117. doi:10.1007/s10072-007-0800-6

17. Lovrencic-Huzian A, Demarin V, Rundek T, Vukovic V (1998) Role of vertebral artery hypoplasia in migraine. Cephalalgia 18:657

18. Chuang YM, Hwang YC, Lin CP, Liu CY (2008) Toward a further elucidation: role of vertebral artery hypoplasia in migraine with aura. Eur Neurol 59:148-151. doi:10.1159/000111878 